\title{
Geographische Nachwuchsforscher in der Schweiz
}

Die folgenden zwei Beiträge sind von jungen Geographinnen und Geographen verfasst, die an dem Symposium am 8 . Oktober 1988 in Lausanne teilgenommen haben.

NUTRIENT CHARGES IN WATER AND EARTHWORM PRODUCTS OF A SWISS JURA MOUNTAIN DRAINAGE BASIN

\section{Introduction}

The present study is part of a comprehensive research work based on a geoand bioecological systems approach. The field studies were performed in a catchment-area of $2 \mathrm{~km}^{2}$ (elevation: 600 $1200 \mathrm{~m} \mathrm{a.s.1.).} \mathrm{The} \mathrm{area} \mathrm{is} \mathrm{covered}$ by manured pastures, meadows, and $\mathrm{mi-}$ xed forests in equal parts. The substrates consist of carbonates, limestones, and clays covered mainly by residuals and/or soils (EGGENBERGER 1986). Water is an important transporting and reacting agent (see also TRUDGILL 1986). Nutrient charges (i.e. net rainfall or biomass * concentration) in precipitation, soil water, earthworm casts and tissues were considered.

Earthworms influence storage and turnover of soil nutrients (LEE 1985). Their body tissues and casts contain high concentrations of readily available (lactate soluble) plant nutrients. At four sites sampled for the present investigation, nine species were found. Their biomass ranged from 48 to 223 $\mathrm{g} * \mathrm{~m}^{-2}$ and their total cast production from 26 to $1094 \mathrm{~g} * \mathrm{~m}^{-2}$. For earthworm sampling and analysis methods see GLASSTETTER und LESER 1987; GLASSTETTER 1988 .

\section{Results and conclusions}

Fig. 1 to 4 show some aspects of nutrient input and storage at four representative sites: two fat meadows and two fir-beech forests. The differentiation of the nutrient input in precipitation is mainly due to interception and leaching processes in the forest canopy. The soil water quality, however, depends on the solubility of the mineral phase (e.g. availability of cations and acid neutralization capacity), the soil water movements (e.g. interflow), and the residence time of water in soil.

The important nutrient storage capacity of the soil fauna, however, is essentially due to the high earthworm biomass and the resulting considerable earthworm cast production. It also depends on the nutrient concentration in the topsoil that, in turn, is influenced by soil type and land use (vegetation cover and manuring).

Wasser und Regenwürmer als stoffträger in einem Einzugsgebiet des Faltenjura

Vorgestellt wird eine Auswahl von Ergebnissen einer geo- und bioökologischen Untersuchung im Faltenjura. Während der Regenwurmaktivitäts-Periode im Frühjahr wurden Stofffrachten in Niederschlag, Bodenwasser, Regenwurmlosung und -körpergewebe ermittelt. Am Beispiel von vier Messstandorten unterschiedlicher Höhenlage und Nutzung wird deutlich, dass die Differenzierung des Stoffeintrags mit dem Niederschlag in erster Linie durch das Kronendach der Wälder (Interzeption, Leaching) zuzustande kommt. Die Bodenwasserqualität wird vor allem durch Eigenschaften der Mineralphasen wie z.B. der Kationenaustauschkapazität bestimmt. Eine wichtige Rolle spielen auch die Bodenwasserbewegungen, insbesondere Interflow.

Die hohe Nährstofffracht in biotischen Kompartimenten der untersuchten Geosysteme ergibt sich durch die dominierende Regenwurmbiomasse und ihre grosse Losungsproduktion. Ausschlaggebend ist dabei die Nährstoffkonzentration im Oberboden, die u.a. von Bodentyp und Landnutzung beeinflusst wird.

Christa Eggenberger und Michèle Glasstetter, Research group Physical Geography and Geoecology, University of Basel, Klingelbergstr. 16. 


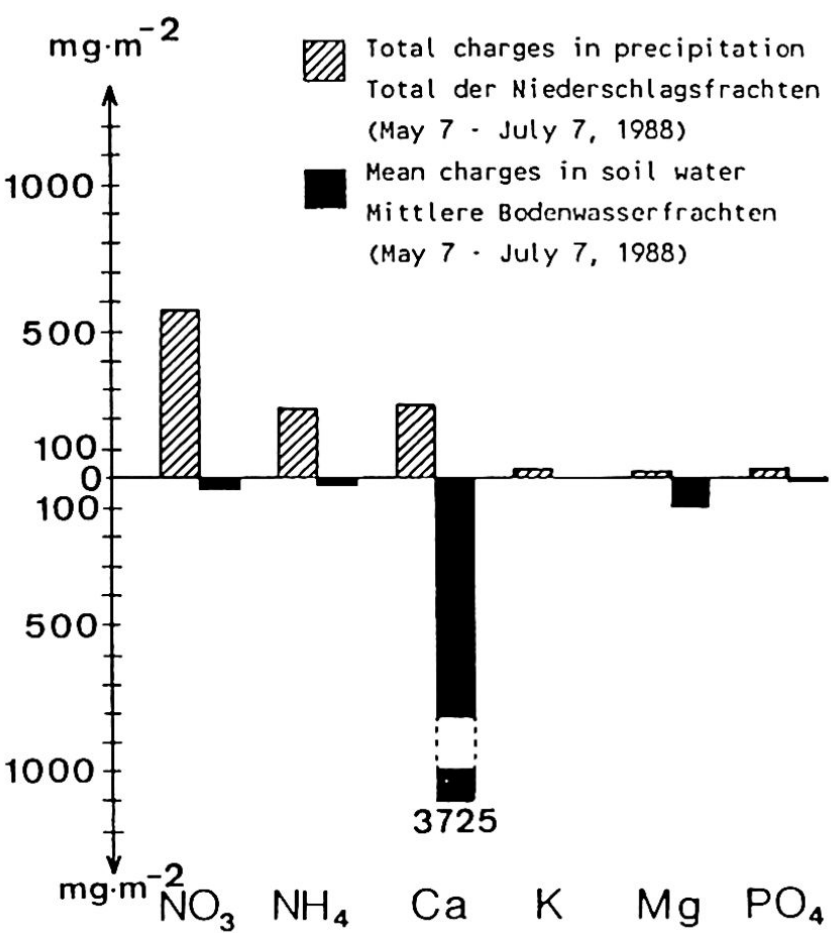

Fig. I Fat meadows/Fettwiesen

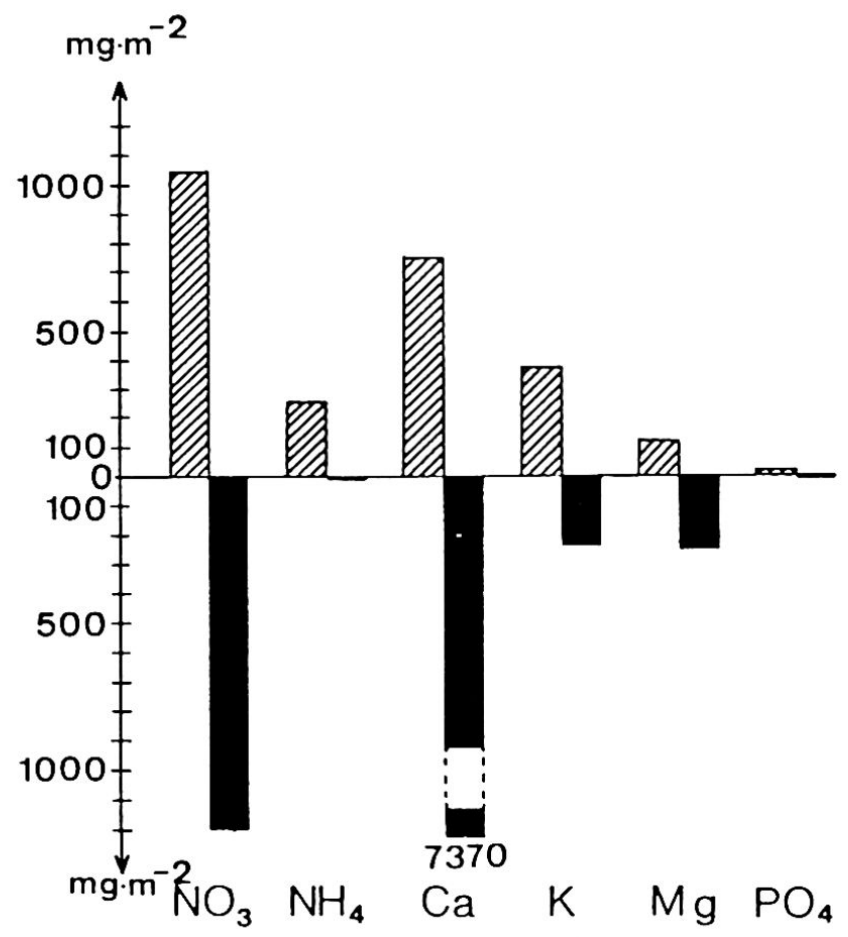

Fig.2 Mixed forests/Mischwälder

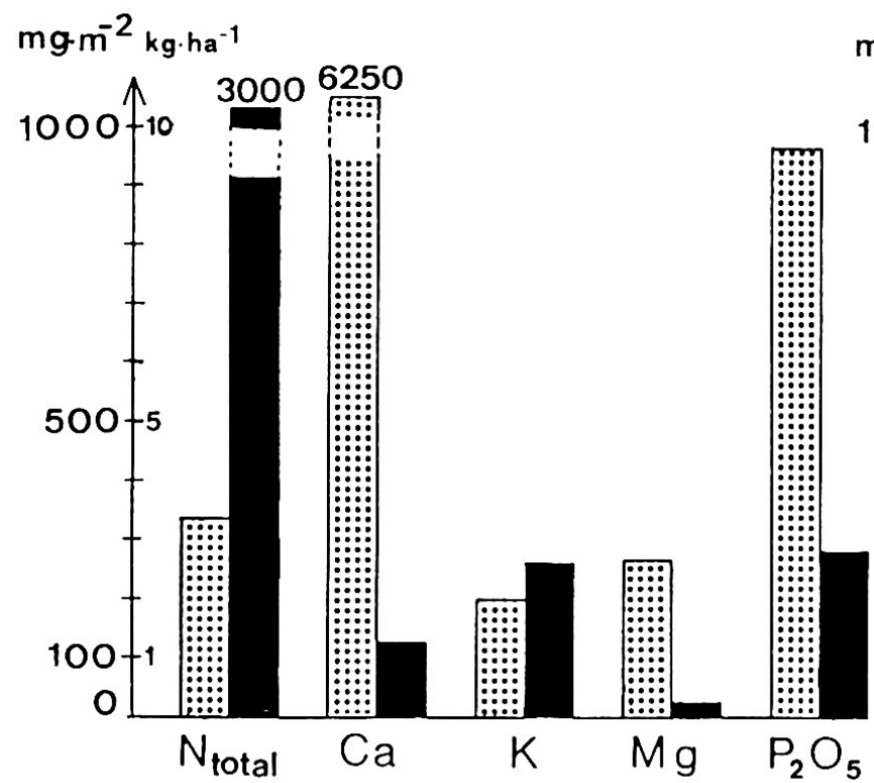

Fig. 3 Fat meadows/Fettwiesen

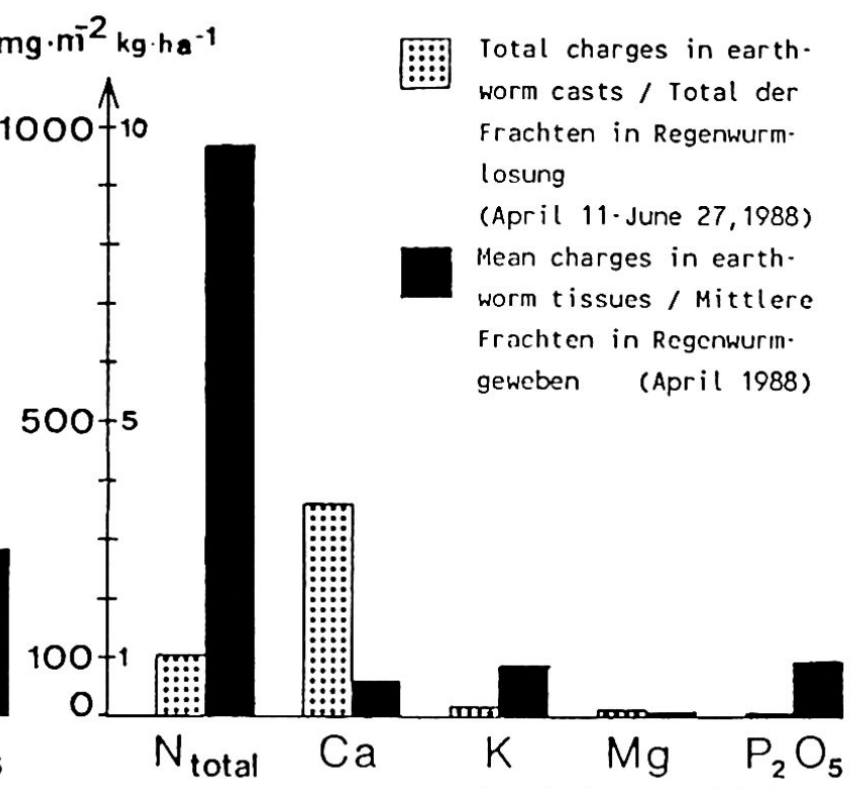

Fig.4 Mixed forests/Mischwälder

\section{References:}

EGGENBERGER, C. (1986): Meso- und Mikroklima eines Einzugsgebietes im Faltenjura. In: Regio Basiliensis 27, 211220.

GLASSTETTER, M. (1988): Die Bodenfauna als Nährstoffspeicher in Geoökosystemen des Tafel- und Faltenjura. In: Bull. Bodenkunkl. Ges. Schweiz 12, 191-194. GLASSTETTER, M. und LESER, H. (1987): Arbeitsbericht über vergleichende Bo-
denfauna-Untersuchungen im Jura als Beitrag zur Erforschung biotischer Kompartimente in Geoökosystemen. In: Regio Basiliensis 28, 183-197.

LEE, K.E. (1985): Earthworms. Their ecology and relationships with soil and land use. Sydney, 409pp.

TRUDGILL, S.T. (ed), (1986): Solute processes. A Wiley-Interscience Publication. New York, 512 pp. 
MODELLIERUNG DER RÄUMLICHEN VERTEILUNG VON PFLANZENGESELLSCHAFTEN AUF DER BASIS ABIOTISCHER FAKTOREN*

Vegetationskarten geben die räumliche Verteilung der vom Standort abhängigen Vegetationstypen in der Landschaft wieder. Diese Karten haben in letzter zeit zunehmend an Bedeutung zur Dokumentation von Veränderungen der ökologischen Situation in der Landschaft gewonnen. Mit $\mathrm{Hilfe}$ eines auf der quantitativen Beschreibung der Standortsansprüche der Vegetation beruhenden Modells ist es möglich, Veränderungen der Vegetation aufgrund von geplanten oder erwarteten Anderungen der Standortsbedingungen vorherzusagen.

Das hier vorgestellte Modell wurde für das MAB-Testgebiet Davos entwickelt. Es umfasst einen etwa $100 \mathrm{~km}^{2}$ grossen Ausschnitt der Landschaft Davos vom subalpinen bis zum nivalen Vegetationsgürtel auf unterschiedlichem Gestein und mit verschiedener Nutzung. Die Standortsdaten wurden im Schweizer MABProjekt Davos erhoben und als Rasterfile mit $50 \mathrm{~m}$ Rasterweite in einem Geographischen Informationssystem gespeichert (Wildi, Ewald 1986). Das Vegetationsmodell ist ein Teil des Landschaftsmodells Davos (Binz, Wildi 1988). Mit Hilfe eines Bayes Klassifikators können metrische, ordinale und kathegorische Variablen verknüpft werden (Fischer 1989). Das Ergebnis ist ein Vektor mit den Schätzungen der multivariaten bedingten Wahrscheinlichkeiten des Auftretens aller im Testgebiet vorkommenden Vegetationstypen. Der Vegetationstyp mit der grössten Auftretenswahrscheinlichkeit ist das Ergebnis der Simulation. Werden diese Berechnungen für jeden Rasterpunkt ausgeführt, erhält man eine flächendeckende Vegetationskarte. Die simulierte Vegetationskarte stimmt in 70 \% aller Rasterpunkte mit dem erhobenen Vegetationstyp und

* eine ausführliche Version des Vortrags erscheint in der Zeitschrift COENOSES in über 90 \% zumindest mit einem ähnlichen Vegetationstyp überein.

Ein solches Modell kann z.B. zur Prognose der Auswirkungen von Änderungen in der Landnutzung oder von globalen Klimaveränderungen auf die Vegetation eingesetzt werden. Karten der Auftretenswahrscheinlichkeit einzelner Pflanzengesellschaften können als Planungsgrundlage bei Wiederaufforstungen und bei der Anlage von Ersatz- und Ausgleichbiotopen dienen. Auch als Kartierhilfsmittel sind derartige Modelle einsetzbar, da punktförmige Information über die Vegetation in die Fläche interpoliert werden kann. Mit zunehmendem Einsatz digitaler Datenverarbeitung in der Kartographie, der auch auf dem Symposium der ASG in Lausanne deutlich wurde, ist $\mathrm{zu}$ hoffen, dass in Zukunft die nötigen digitalen Basiskarten für einen breiten Einsatz in der Praxis zur Verfügung stehen werden.

\section{Literatur}

BINZ, H.R., WILDI,O. (1988): Das Simulationsmodell MaB-Davos. Schlussbericht Schweiz. MaB-Programm Nr. 33, Bundesamt für Umweltschutz, Bern.

FISCHER, H.S. (1989): Simulating the distribution of plant communities in an alpine landscape. COENOSES (in Druck)

WILDI, O., EWALD, K. (1986): Der Naturraum und dessen Nutzung im alpinen Tourismusgebiet von Davos. Ergebnisse des MaB-Projektes Davos. Eidg. Anst. forstl. Versuchswes., Ber. Nr. 289, Birmensdorf.
Hagen S. Fischer, Diplom-Biologe, Geographisches Institut der ETH, winterthurerstr. 190, 8057 zürich 